\title{
Management of bronchial carcinoma by segmental resection
}

\author{
B. T. LE ROUX \\ Thoracic Unit, Edinburgh ${ }^{1}$
}

Of 4,000 patients with bronchial carcinoma, a small peripheral tumour in 17 was managed by segmental resection because the lesion palpated at thoracotomy was thought to be tuberculous, because the segment in which the tumour lay was separated from adjacent lung by an anatomically complete fissure, or to avoid unnecessary limitation of an already diminished respiratory reserve. One patient died as a direct result of operation; eight died from metastases or recurrence of tumour one to seven years after operation; three died from non-metastatic causes; and five are alive five to eight years after operation. From this small selected group of patients the results of management of bronchial carcinoma by segmental resection seem no worse than after more extensive resections for small tumours.

Of 4,000 patients with bronchial carcinoma managed in the Regional Thoracic Unit in Edinburgh between 1949 and $1963,1,783$ patients $(44 \cdot 6 \%)$ were submitted to exploratory thoracotomy, and some form of pulmonary resaction was completed in 1,464 patients $(36 \cdot 6 \%)$. Surgical management was precluded in 1,834 patients $(45.9 \%)$ because of clinical, radiographic, fluoroscopic or bronchoscopic evidence of dissemination of tumour, and in 383 patients $(9 \cdot 5 \%)$ because of evidence of unsuitability for surgical management other than metastases, or because they declined operation. Of those managed surgically, the resection undertaken was a segmental resection in 17 patients.

The purpose of this paper is to give the reasons for having undertaken so limited a resection in these few patients and to assess the results. At no time in the period under discussion was segmental resection regarded as an acceptable alternative to lobectomy in the management of established bronchial carcinoma, and the circumstances in which segmental resection was undertaken in these 17 patients were unusual.

Of the 17 patients, eight were older than 65 years-four of these were over 70 years. The youngest patient was aged 39 years. All but one were men. In all, the tumour was peripheral in type and the appearances at bronchoscopy were normal. The tumour was in the right lung in eight and in the left lung in nine patients. In seven

1Present address: Thoracic Unit, Wentworth Hospital, Durban patients the segment resected was an apical sege ment of an upper lobe and in another seven a apical segment of a lower lobe; in two, the lingular segment was resected and in one, a segment of thes middle lobe. Presentation in five symptomles patients was with an abnormal chest radiograph made for routine purposes, and in one patien? presentation was with the symptoms of hypers trophic pulmonary osteoarthropathy. The perf pheral spherical pulmonary shadow in three patients measured $1 \mathrm{~cm}$ in transverse diameter; i] seven patients the peripheral pulmonary shadow measured less than $1.5 \mathrm{~cm}$, and in five other patients was less than $2 \mathrm{~cm}$ in transverse diametes. in only two patients was the peripheral pulmonaris opacity greater than $2 \mathrm{~cm}$ in diameter. In 1 委 patients the tumour was squamous in type, in ons undifferentiated, and in four patients the tumour was an adenocarcinoma. In three patients resero tion of the segments included a healed tuberculous lesion in very close proximity to the tumour; ian two patients there were two separate tumours ino the same segment, one smaller than the othen not separately recognized tomographically, and insterpreted at operation as a tuberculous focus with a satellite lesion in close proximity; in two patients the tumour was of calcific hardness.

The reasons for management of bronchial carcinoma by segmental resection in these 18 patients were: (a) interpretation by palpation of the lesion in seven patients as tuberculous on the grounds of hardness, related scarring or mult? plicity of nodules; $(b)$ separation of the resected 
segment from neighbouring segments by an anatomically complete fissure in five patients, in four of whom the apical segment of a lower lobe and in one of whom the lingular segment was resected; in these the lesion was recognized as a probable carcinoma, but it was judged as effective to manage the tumour by segmental resection as by lobectomy; (c) severe limitation of respiratory reserve in five patients, in whom thoracotomy was undertaken with a view to doing the most limited resection possible to establish the diagnosis of carcinoma, and in whom it had been decided, before operation, that a resection greater than lobectomy would not be undertaken.

Of the 17 patients in whom bronchial carcinoma was managed by segmental resection, one died from respiratory failure as a direct result of operation-one of the five in whom respiratory function was severely limited. Five patients were alive - two of these five years after segmental resection, one six years and two eight years after operation-and in three of these five, segmental resection was undertaken because of completeness of anatomical separation of the resected segment from adjacent lung. Of the five long survivors, the tumour in two was an adenocarcinoma and in three a squamous carcinoma. Two still work as miners at the coal-face. Eleven patients have died, eight of these from metastases and three from causes apparently unrelated to bronchial carcinoma. Two patients died from metastases within a year of operation, four in the third postoperative year, one in the fifth, and one in the seventh postoperative year.

Two patients died from coronary artery disease two years after operation and one died 18 months after operation in the following circumstances: left apical segmental resection had been undertaken in the belief that the lesion was tuberculous (Fig. 1) and when the histological report of carcinoma was received, further treatment was by radiotherapy to the area from which the segment had been removed; a radiograph made soon after operation was accepted as normal for the procedure undertaken (Fig. 2); nine months later the patient became ill with severe pulmonary infection and either an abscess in the left upper lobe or a localized left upper empyema (Fig. 3); he failed to improve on conservative management (Fig. 4) and the loculus of pus was drained anteriorly by rib resection (Fig. 5); a large pulmonary slough was removed at this operation, and subsequent bronchography demonstrated a communication between the left upper bronchus and the large, static left upper pleural space with

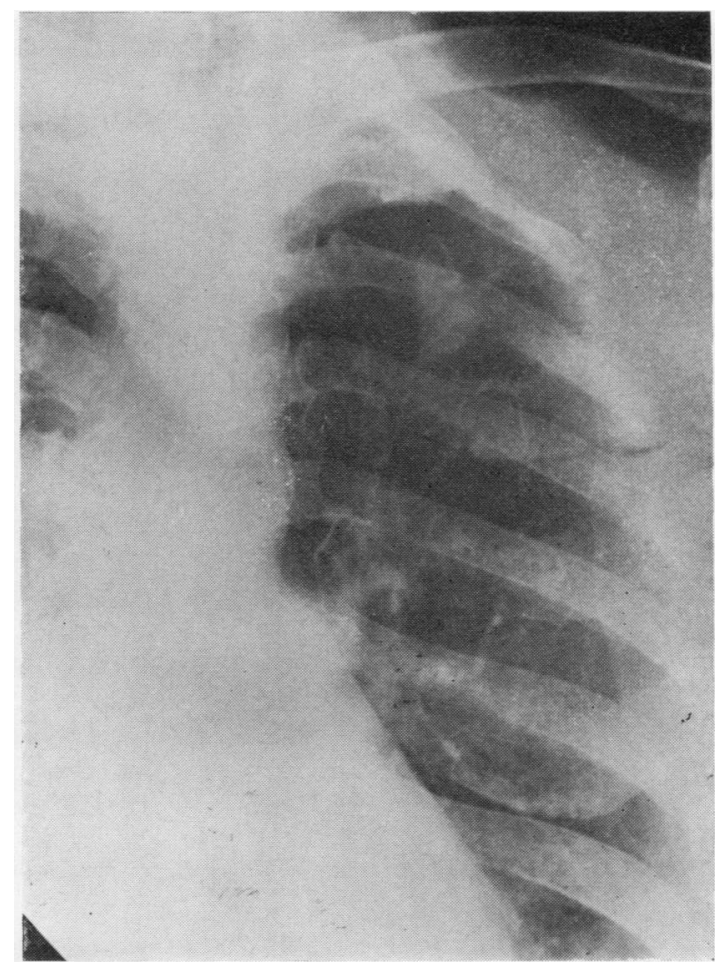

FIG. 1. Pear-shaped opacity in left upper lobe, thought to be tuberculous.

its anterior parietal drain; radiotherapy was judged responsible for sphacelation of most of what remained of the left upper lobe after apical segmental resection; continued deterioration in health and recurrent contralateral aspiration pneumonia prompted injudicious closure of the left upper space by thoracoplasty, in convalescence from which he died; metastases were not found at necropsy. Radiotherapy was not used in other patients in this group of 17 until there was evidence of recurrence of tumour.

From the small number of segmental resections undertaken it can only be said that this operation, undertaken in the management of bronchial carcinoma, is followed by long survival in a proportion of patients not noticeably different from that which follows resection of greater extent for tumours of the same relatively small size. Since in none of the patients managed by segmental resection were hilar glands resected for histological examination, and since none of the operative notes contains specific comments on the presence or absence of such glands, it is not possible to 


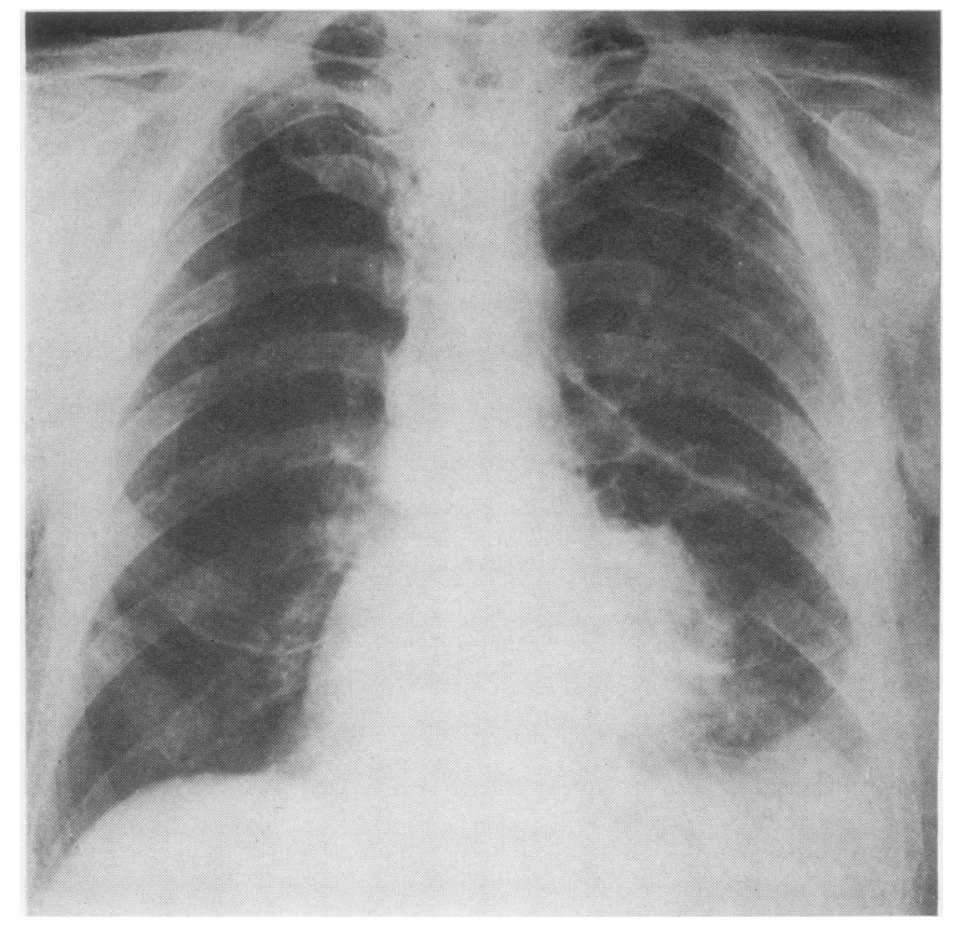

FIG. 2. An early postoperative posteraanterior radiograph made after le apical segmental resection.

FIG. 3. Postero-anterior chest radiograph made nine months after segmental resection and irradiation, in which is shown the left upper cavity, thought to be either a lung abscess or an empyema.

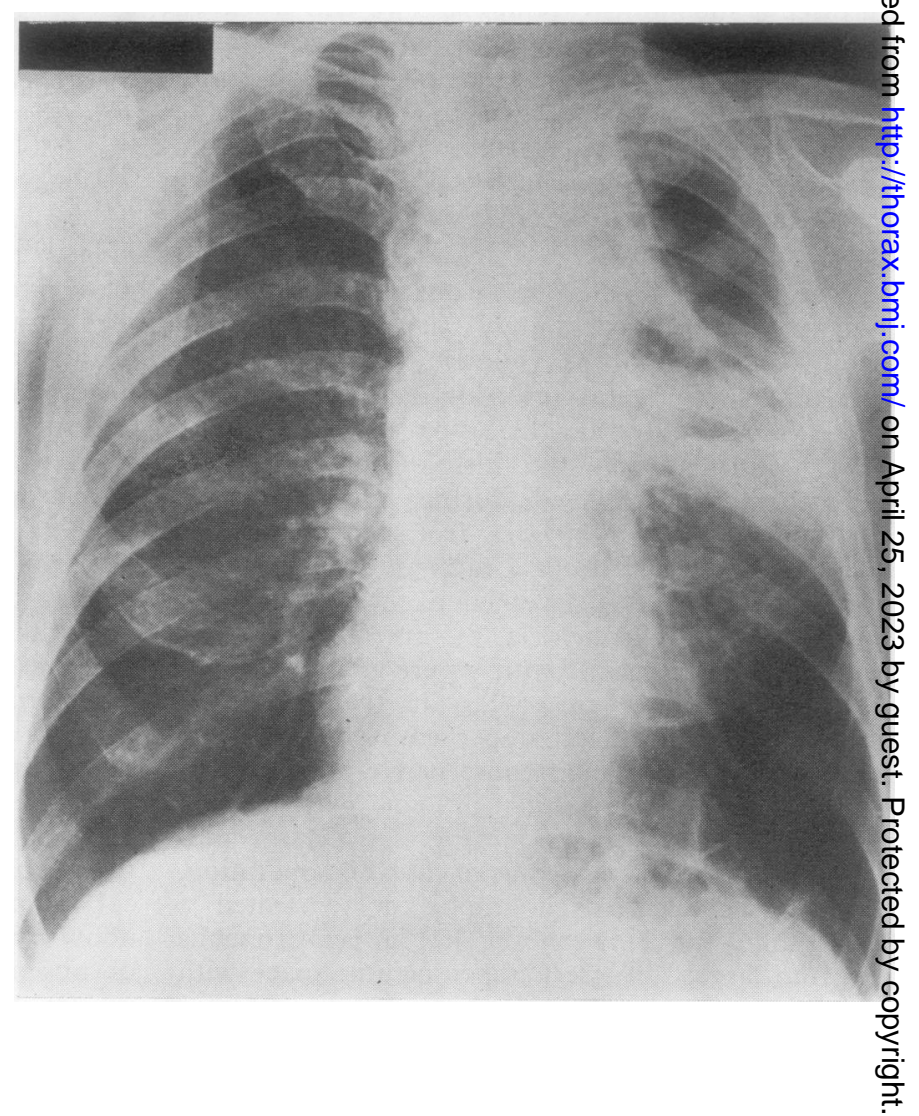




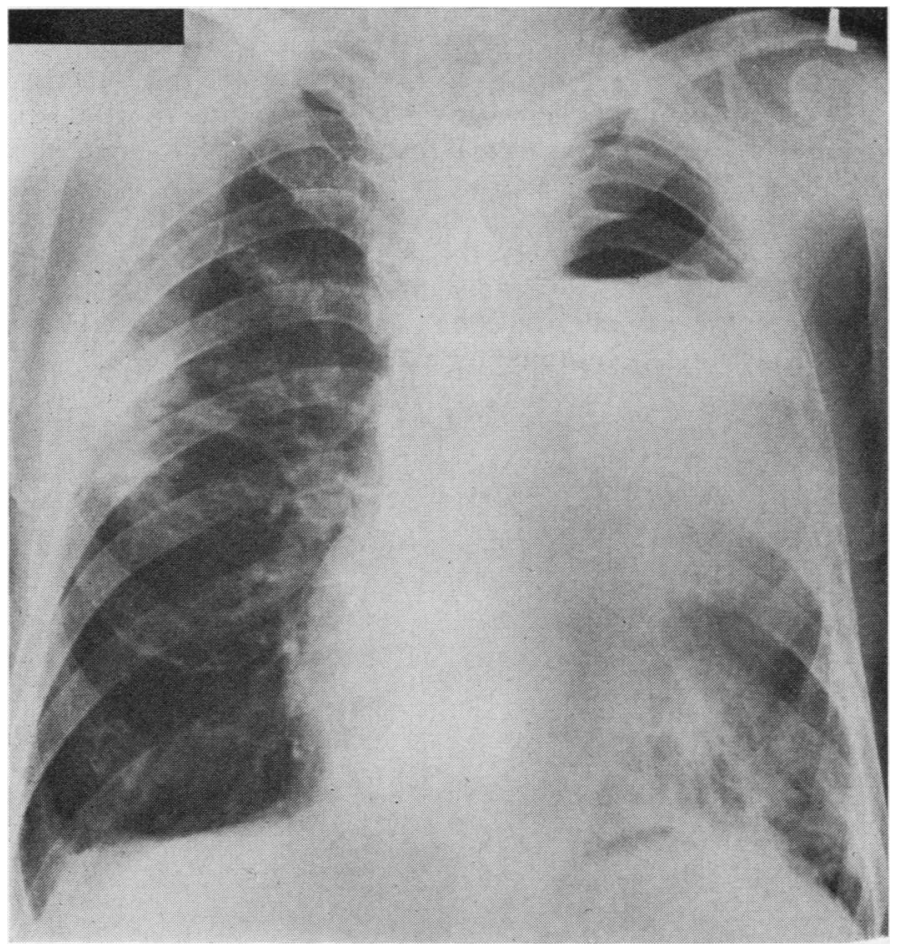

FIG. 4. Conservative management, with physiotherapy and chemotherapy, failed to influence the left upper cavitated lesion. Postero-anterior chest radiograph shows that the cavity retained liquid and that contralateral spill had occurred.

FIG. 5. Antero-posterior radiograph made after left anterior rib resection.

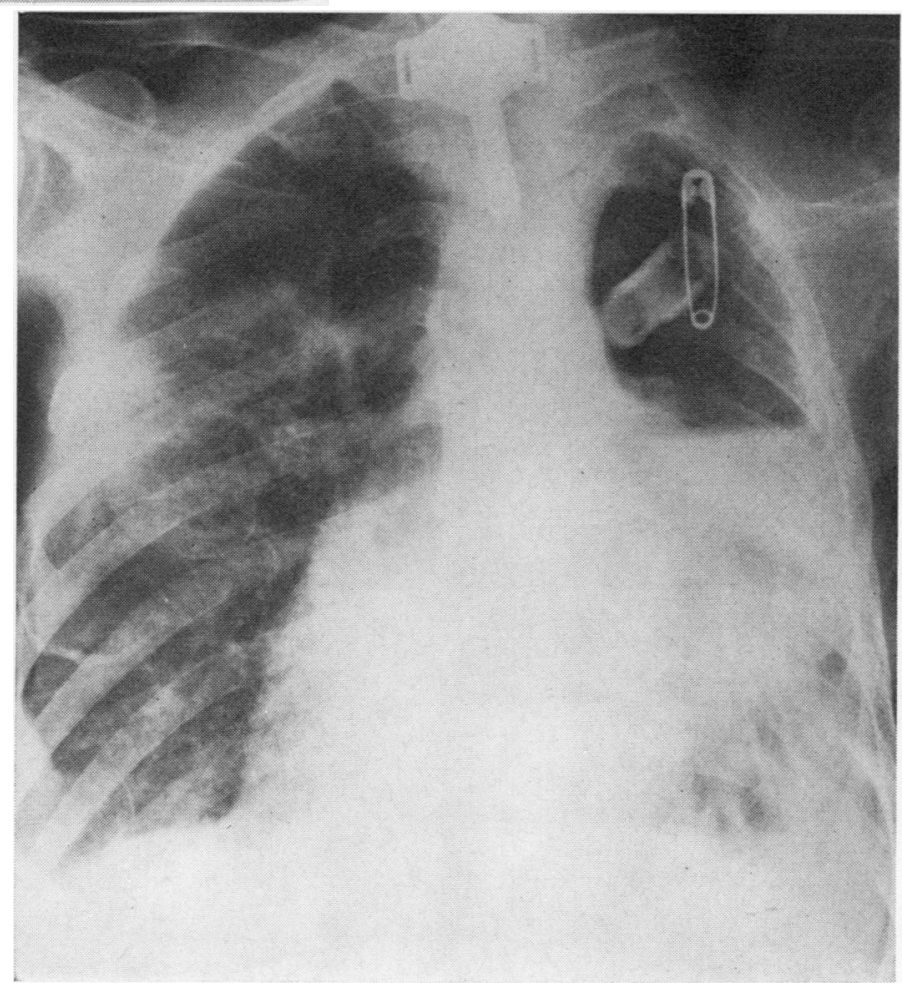


relate survival to the absence of hilar glandular invasion, although this remains a reasonable assumption. In three patients who died with metastases there was radiographic evidence before death, and histological evidence after necropsy, of local recurrence of tumour. In one of these patients local recurrence became radiographically evident in the fourth year after segmental resection for squamous carcinoma, and death was delayed a further three years by irradiation. Death was related to distant metastases or mediastinal growth of tumour in the remainder who died with metastases-hepatic metastases without local recurrence in two, obstruction of the superior vena cava in two, and interruption of the left recurrent laryngeal nerve in one. Survival time and the pattern of emergence of metastatic tumour or recurrence of tumour at the site of resection or in the mediastinum are, therefore, also not noticeably different in those managed by segmental resection from those which follow resestion of greater extent. 